\title{
La dialéctica temporal de la observación empirica en las ciencias de la sociedad ${ }^{1}$
}

The dialectic of time of empirical observation in sciences of society

\author{
JAVIER CALlEJO \\ Centro de Investigaciones Sociológicas \\ jcallejo@cis.es (ESPAÑA)
}

Recibido: 06.02.2011

Aceptado: 25.12.2011

Finalement le temps objectif, c'est le temps maximum: c'est celui qui contient tous les instants. Il est fait de l'ensemble dense des actes du Créateur

(Gaston Bachelard, en L'intuition de l'instant)

\section{RESUMEN}

A partir de un diálogo entre las obras de autores tan distantes como Michel de Certeau y Niklas Luhmann, se realiza un trabajo de proyección de los conceptos de estrategia y táctica en el análisis de las distintas dimensiones temporales presentes en la observación empírica de las ciencias sociales. Aparecen dos temporalidades prácticamente opuestas, la de la duración de las estrategias y la del instante de las tácticas. La temporalidad propia del sistema social de la ciencia y la temporalidad del encuentro entre este sistema social y los sistemas sociales observados. Dos temporalidades que parecen negarse recíprocamente y que, sin embargo, en su relación, construyen la observación empírica.

\section{PALABRAS CLAVE}

temporalidad, sociología del tiempo, estrategia, táctica.

1 Quisiera agradecer los comentarios recibidos al original por los evaluadores anónimos del artículo, por su detalle, profundidad y fuerza para motivar una nueva reflexión. Sin duda, el implícito diálogo con ellos ha mejorado el producto final. 


\begin{abstract}
From a dialogue between the works of Michel de Certeau and Niklas Luhmann, this work offers a projection of the concepts of strategy and tactics in the analysis of different time dimensions present in the empirical observation of the social sciences. Temporalities are two almost opposite that of the duration of the strategies and the instant of the tactics. The temporality of the social system of science and the temporality of the encounter between this social system and social systems observed. Two temporalities interact and seem to refuse, however, in their relationship, produce empirical observation.
\end{abstract}

\title{
KEYWORDS
}

temporality, sociology of time, strategy, tactics.

\section{INTRODUCCIÓN}

El análisis crítico de los procesos de la observación empírica en general y especialmente de la observación empírica sociológica ha estado más centrado en la determinación de ésta sobre lo observado, hasta entrar en perspectivas constructivistas o, del otro lado, en la obtención de categorías normativas sobre el quehacer de la observación. Desde la primera perspectiva, se plantea principalmente la observación como acción social (Cicourel, 1982) y se sitúan centralmente conceptos como reactividad, siguiendo a las ciencias físico-químicas y se otorga un papel principal a estudios clásicos, como los experimentos en la fábrica de Hawthorne (Mayo, 1975). Más o menos explícito se encuentra la asunción de que la observación genera efectos en lo observado, en los observados. Desde un punto normativo, como el presente en los manuales de metodología, se llama la atención para que la observación, lo producido por la observación, no determine la observación (los «resultados» de la observación), e incluso se defiende la ventaja de ciertas prácticas de investigación social desde su menor reactividad, en cuanto se las considera poco o nada intrusivas (Webb et al., 1966; Flick, 2004; Banks, 2001; Sutton y Matthew, 2011).

Sin embargo y a pesar de haberse insistido en que la propia situación de observación es una situación genuinamente social (entre otros: Alonso, 1998; Valles, 1997), se tiende a dejar al margen que, más allá o más acá de las determinaciones producidas por una situación siempre experimental —en cuanto salida de la experiencia- de la observación, lo primero que causa es un encuentro entre presentes, por lo tanto, una simultaneidad, una concreción temporal.

La adaptación entre la entidad o sistema observador y la entidad o sistema observado, que tiende a constituirse en un acotado mundo cotidiano, en la observación sociológica se ha planteado críticamente en distintas claves, como la 
lingüística y cognitiva (Cicourel, 1982), asumiendo la posibilidad de tal adaptación, ya sea a partir de una teoría del actor ante los métodos de observación, ya de una teoría del método en la vida cotidiana, como plantea la etnometodología. Pero apenas se ha abordado desde la necesaria adaptación de tiempos.

Lo primero que produce la observación, antes de cualquier resultado, es la simultaneidad de una presencia, de una copresencia, la de quien ocupa la posición de observador y la de quien ocupa la de observado. Es lo que aquí se intenta a través de dos conceptos ampliamente utilizados en la literatura metodológica, como son los de estrategia y táctica (Cea, 1999; Valles, 1997; Delgado, 1994; Douglas, 1976; Layder, 1993; Krisnhaswami, 2004). Especialmente el primero de ellos ha hecho fortuna, hablándose de, por ejemplo, investigación estratégica bajo la influencia de la investigación social aplicada en general y del marketing en particular.

El presente trabajo se ocupa de abordar las implicaciones teóricas de la observación del tiempo de la observación empírica. Para ello, se incrusta en la relación entre sistemas sociales que exige la observación empírica, donde se encuentran el sistema social observador, configurado como sistema social ciencia, y el sistema social observado, siempre distinto salvo en una excepción: cuando el sistema social observado empíricamente es el propio sistema social de la ciencia. De alguna manera, nos incrustamos en tal excepción, si se asume el trabajo como una observación del sistema social ciencia en su relación con los sistemas sociales observados.

La distinción de las distintas temporalidades que actúan en la observación sociológica, lo que asume la hipótesis de la existencia de varias temporalidades y el compromiso analítico de su relación, es un primer paso para enfocar metodológicamente cada una de ellas. En especial, aquella menos abordada y más vinculada a la reactividad, como es la simultaneidad.

\section{EL TIEMPO DE LA OBSERVACIÓN, DESDE LA OBSERVACIÓN EMPÍRICA}

El presente trabajo es la continuación teórica de un trabajo empírico colectivo (Prieto et al., 2009) en el que se profundizaba en las relaciones entre tiempos de trabajo o empleo y otros tiempos, especialmente el dedicado al trabajo doméstico; y de una continua reflexión personal sobre el significado de la temporalidad en la observación empírica.

La investigación empírica referida puso de manifiesto, entre otras cosas:

- La pertinencia de las propuestas teóricas y conceptuales de Michel de Certeau (de Certeau, 1990; de Certeau et al., 1994) al abordar las prácticas cotidianas de los sujetos, de manera que lejos de la coherencia o cierta linealidad, a las que tiende la formalización de su observación, se constituyen de manera cambiante, renovadora, transformándose, en función de las circunstancias. Lejos de poder hablarse de rutinas, el intenso 
trabajo de campo manifestaba un continuo y renovado flujo de acuerdos con los demás y una especie de bricolaje de las distintas prácticas. Algo que también se puso de manifiesto en el estudio colectivo referido (Prieto et al., 2009), como en otros estudios empíricos que, enfocados inicialmente en el tiempo de trabajo, han visto la necesidad de abordar su articulación con los tiempos de otros ámbitos de prácticas, especialmente cuando se aborda el tiempo de trabajo de los sujetos atravesados por posiciones en diversos de esos ámbitos o, como lo vamos a denominar aquí, sistemas sociales, como es el caso de las mujeres (Torns, 2005; Prieto, 2007), donde se requieren arreglos, composiciones, para adaptar y adaptarse a esa pretendida linealidad rutinaria. Pues bien, la observación, que tiende a tomar la perspectiva desde uno sólo de los sistemas sociales, trabajo-empleo o tareas domésticas, tiende a aislarse de los ruidos que deja la relación con el otro sistema social. Sólo desde perspectivas que tienen por horizonte la propia articulación entre sistemas, sus puntos de conexión, que tienden a ser perspectivas que parten de los sujetos como unidad en la que tienen lugar esas prácticas de articulación y esos cruces de sistemas sociales, parece que afloran los ruidos, las dificultades para las componendas y, en definitiva, las quiebras de esa linealidad rutinaria, especialmente subrayada cuando se trata de aproximaciones estadísticas: tiempo dedicado al empleo remunerado, por un lado; tiempo dedicado al trabajo doméstico o cualquier otra práctica, por otro lado. Sin tener en cuenta los choques entre ambos, dejándolo, a lo sumo, en meros cruces estadísticos — como denunciaba Bourdieu (1980)— se pierde la articulación. De aquí la pertinencia de aproximaciones como, entre otras, las de Michel de Certeau y su equipo. Una aproximación que requiere hacer uso de conceptos como estrategias y tácticas, como conceptos en los que se enfrentan las expectativas - trayectorias más o menos imaginarias de poder hacer, casi siempre dentro de uno sólo de los sistemas sociales-y la rugosidad más o menos laberíntica y conflictiva de las trayectorias concretas, de lo que los sujetos hacen en y con su vida cotidiana, lo que al final tiende a desembocar en sujetos quebrados por esa realidad temporalmente quebrada, bastante lejos de una cristalina identidad. Sujetos quebrados que tiende a poner de relieve la literatura de la postmodernidad: un tiempo oscuro (Bauman, 2005) que oscurece o, mejor, disipa sus tiempos y las relaciones entre sus distintos tipos o categorías. Ahora bien, estrategias y tácticas que son conceptos fundamentalmente temporales, puesto que plantean la relación y proyección de los sujetos con el tiempo. Son estrategias y tácticas en el tiempo (Callejo, 2005).

- Estrategias y tácticas en el tiempo, de manera que unas tendían a constituirse en límite de las otras, de manera que parece no tener sentido abordar unas sin tener en cuenta las otras. Es decir, las proyecciones estratégicas se alimentaban o frustraban en función de las tácticas que debían llevar a cabo los sujetos, y las coordinaciones tácticas adquieren 
sentido en función de horizontes estratégicos. Ahora bien, como se ponía de manifiesto en el análisis de los resultados de esa investigación (Callejo, 2005), las proyecciones estratégicas, las posibilidades de alimentar estrategias venían dadas por las condiciones y posiciones que ocupaban las distintas categorías de sujetos — principalmente en función del género: varones y mujeres - en los distintos sistemas sociales y de la relación entre tales sistemas sociales. Así, los varones ocupan un lugar dominante en el sistema social productivo, especialmente en el mercado laboral; pero, sobre todo, apenas soportan el ruido de la mediación y la vinculación con el sistema social reproductivo. Ello hace que requieran menos ejercicio táctico y dispongan de mayor margen estratégico en ese sistema social. Por el contrario, las mujeres, desde la perspectiva del sistema productivo, son condenadas a soportar más ruido y mediaciones del sistema social reproductivo y, por lo tanto, mayores exigencias del esfuerzo táctico y menor disponibilidad estratégica para la movilidad en el sistema social productivo. Lo que interesa destacar aquí son otras proyecciones teóricas de este análisis, relacionadas potencialmente con la teoría de sistemas, como es que la mayor o menor presencia de decisiones tácticas o, en general, de tácticas, dependerá del tipo de relación que se establezca entre sistemas. Es decir, intentando ser más fiel a la concepción de la teoría de sistemas, en la medida que un sistema se convierta en el entorno del otro. De hecho, a partir del material empírico recogido, podría decirse que para los varones, el sistema social reproductivo tiende a convertirse en el entorno del sistema social productivo; $y$, para las mujeres, el sistema social productivo se convierte en el entorno del sistema social reproductivo. Pero ello sería focalizar el análisis en las propias categorías de sujetos, lo que parece impropio de la propia concepción sistémica. Por ello, nos quedamos en la constatación de que una mayor relación entre sistemas sociales tiende a generar más movimientos tácticos, lo que es situar el concepto de táctica en ese lugar de enfrentamiento entre sistemas. En la siempre fluctuante línea entre sistemas. ¿Y las estrategias? Aún centrándonos en nuestro trabajo empírico de partida, la disponibilidad de margen para las estrategias se fundamentaba en la menor presencia de relación o mediación que el sistema social productivo tenía con el sistema reproductivo. De hecho, también podría invertirse el análisis: la mayor disponibilidad de estrategias en el sistema social reproductivo deriva de una menor relación o mediación con el sistema social productivo; así, por ejemplo: las amas de casa se organizan mejor y planifican mejor, para ellas y el conjunto de la familia, en la medida que no tienen que atender a un empleo; permitiendo, de paso, que los otros sujetos familiares viesen menos mediado los sistemas productivos (empleo, estudios) por el sistema social reproductivo. Si las tácticas parecen situarse en los encuentros entre sistemas sociales, las estrategias parecen hacerlo en el aislamiento entre sistemas sociales. 
Por otro lado, hay que señalar la relativa debilidad de la oposición perspectiva metodológica sincrónica frente a perspectiva metodológica diacrónica, radicada en el estructuralismo lingüístico (Saussure, 1983)² para la observación sociológica empírica. Especialmente lo diacrónico se revela como sucesión de sincronías y, a su vez, lo sincrónico tiende a aparecer menos como una puesta en común de tiempos - a lo que parece referirse el término sincronización-que como un esfuerzo por denegar el tiempo, por hacer desaparecer la temporalidad de la observación metodológica y, por lo tanto, de la investigación. En el mejor de los casos, la oposición sólo se refería a la temporalidad del objeto. En la denominada perspectiva sincrónica, la temporalidad del objeto tendía a cero. De aquí el extendido y exitoso uso de metáforas como el de la fotografía. Se paraba el tiempo del objeto observado, lo que exigía que la propia observación tomase las características de lo instantáneo, de una práctica sin duración. Del instante. En la denominada perspectiva diacrónica, se establecía la serie de sucesiones entre las distintas observaciones sincrónicas. Por lo tanto, entre $\mathrm{t}_{1}=0 \mathrm{y} \mathrm{t}_{2}=0 \mathrm{se}$ proyectaba el tiempo, con dos asunciones: la observación no tenía duración, no conllevaba tiempo; y el tiempo del objeto observado se establecía como la diferencia entre $\mathrm{t}_{2} \mathrm{y} \mathrm{t}_{1}$, sin que se registrase la experiencia de la propia temporalidad.

Además, los conceptos estrategia y táctica se han incrustado con éxito en la literatura metodológica (Douglas, 1976; Layder, 1993; Valles, 1997). Ahora bien, lo han hecho desprovistos de tiempo, de temporalidad, y en la mayor parte de los casos adscritos a las potenciales decisiones metodológicas de un hipotético investigador. Lo que en nuestra observación empírica está trufado de tiempo, parece desaparecer de éstos en la reflexión metodológica más normativa.

\section{UNA INTERPRETACIÓN SISTÉMICA DE MICHEL DE CERTEAU: LA VIDA COTIDIANA (Y EL MUNDO DE VIDA) COMO ENCUENTRO ENTRE SISTEMAS SOCIALES (A PESAR DE LUHMANN)}

La conexión entre prácticas y, por lo tanto, la conexión entre tiempos ha tendido a quedar como una caja negra en la sociología o, al menos, como una evidencia oscura. La conciencia de nuestra experiencia vital nos dice que, al menos, el tiempo de una práctica condiciona el tiempo de otras prácticas, teniendo en cuenta la humana sujeción a un tiempo finito. La sociología del tiempo se ha extendido principalmente más en las distribuciones sociales del tiempo, que en las conexiones entre tiempos, que son conexiones entre prácticas y, como consecuencia, conexiones entre actores y sistemas. Es en la conexión de prácticas donde se sitúa de Certeau, de aquí la pertinencia de su referencia.

Michel de Certeau, de la mano de Clausewitz, hace centrales (Giard,

\footnotetext{
2 Remarcando la perspectiva diacrónica en Saussure, véase López Martínez y Hernández Sánchez (2002).
} 
1990:XII) los conceptos de estrategia y táctica frente a la estabilidad de cierta mirada estructuralista, citando expresamente a Levi-Strauss y Greimas, y asumiéndose deudor en el trabajo sobre estos conceptos de las aportaciones prácticamente simultáneas a su propia producción- del Foucault de Vigilar y castigar (Foucault, 1978) y, sobre todo, del Bourdieu de El sentido práctico (Bourdieu, 1988) .

Para de Certeau, la estrategia es: «el cálculo de las relaciones de fuerzas que son posibles a partir del momento en el que un sujeto de querer y de poder es aislable de un "entorno". Postula un lugar susceptible de quedar circunscrito como una propiedad y por lo tanto de servir de base para una gestión de sus relaciones con una exterioridad distinta. La racionalidad política, económica o científica se construyen sobre este modelo estratégico» (de Certeau, 1990: XLVI y 59). Una definición que se introduce en el texto en pleno diálogo con la obra de Bourdieu, reconociéndola y distanciándose con matices. Para Bourdieu, la estrategia está relacionada con las reglas del campo social y la maximización de lógicas y capitales (Bourdieu, 2002), que son los núcleos de las lógicas en los campos. De Certeau subraya el concepto de sujeto; pero aquí, retomamos aún más dos de las características de su estrategia, como es el aislamiento del entorno y la racionalidad. ¿Qué es el aislamiento del entorno en de Certeau? Parece que sólo cabe entenderlo como producto imaginario.

Como aislamiento del entorno, el concepto de estrategia del autor de origen francés nos introduce en el lugar relativo de tal sujeto. Ha de hacerse la ficción de aislarlo del entorno. Un sujeto nunca es aislable del entorno. Sin embargo, los sistemas sociales parten de su distinción del entorno. Donde es imposible el sujeto de la estrategia, nace la estrategia para el sistema. Y, sobre todo, un ejercicio de racionalidad, un gesto cartesiano (de Certeau, 1990:59). ¿Racionalidad, de qué? Racionalidad del sistema. De Certeau opta por poner una voluntad, un querer, en la estrategia, haciendo así al sujeto soporte de la misma y, en términos temporales, de la propia duración. La voluntad requiere de la duración.

De Certeau seguirá completando el contenido del concepto de estrategia. Es imaginaria y global. Como imaginaria, es proyección y, sobre todo, es del sentido. Se plantea en un sentido. La acción estratégica, en la medida que quepa asimilar los momentos incluidos en una práctica como acción ${ }^{4}$, es una acción con sentido. Un sentido que reconstruye y produce los actos en un álgebra de los actos, en términos del poeta Paul Valéry.

Es una acción con un horizonte de totalidad, de globalidad. ¿Qué globalidad? ¿es la globalidad del sujeto entendida como identidad? Como se verá al presentar la concepción de táctica, la globalidad es el otro. El Otro, con mayúsculas, del

\footnotetext{
3 Sobre el uso de los conceptos de estrategia y táctica, en buena parte éste subsumido en el concepto de habitus, a partir de la obra de Bourdieu, véase Callejo (1995:228 y ss., 2004).

${ }^{4}$ Lo cual no deja de traer problemas: ¿cuántos momentos tiene una acción? ¿cabe asimilar momento y acción? La acción, que se tiene a presentar como concreción del sentido, parece carecer de contenido temporal, más allá de su proyección histórica.
} 
propio de Certeau (1993) en la senda lacaniana. Es la totalidad de la alteridad, lo que habrá que tomar como el conjunto de la sociedad.

El concepto de táctica se configura en de Certeau como la cara opuesta - y, en muchos casos oculta, tal vez conceptualmente débil- de la estrategia: «cálculo que no cuenta con ninguna propiedad, ni por lo tanto con una frontera que distingue al otro como una totalidad visible. La táctica no tiene por lugar más que el del otro. Se le insinúa fragmentariamente, sin tomarle enteramente, sin poder tomar distancia con respecto a él [...] Al contrario del hecho de su no lugar, la táctica depende del tiempo» (de Certeau, 1990: XLVI). Caracterización negativa de la táctica, a partir de lo que no es, salvo que es tiempo y, sobre todo, tiempo-con-el-otro. Otro concreto, en oposición al otro global de la estrategia. Incluso parece que se borra el sujeto, a favor del otro, de la alteridad. Ya no hay voluntad, ni deseo. La táctica parece desbordar al sujeto, ir más allá de sus fronteras. Es la reacción —incluso corporal- del actor ante las circunstancias. Fuera del sujeto y fuera del sistema, porque está en la comunicación entre sistemas. Nos habla de su inserción en las interferencias (de Certeau, 1996:51). La táctica se sitúa en flujos de comunicación reactivos y esfuerzos de adaptación a la situación.

Como hizo con el concepto de estrategia, hace lo propio con el de táctica, llenándolo de contenidos. De la misma definición, cabe deducir que las tácticas son del encuentro, con el otro, y, por lo tanto, locales, espacialmente. Desde el punto de vista temporal, son del instante (de Certeau, 1990:63). Desde la perspectiva del comportamiento de los sujetos, las tácticas niegan al sujeto, son de la simulación (de Certeau, 1990:42), que es el dejar de ser sujetos o, al menos, una forma de ser sujetos que deniega su posición de sujeto. Simular es así también la táctica de estar en comunicación con otro sistema social, sin entrar en él, pues es imposible cambiar de sistema. Se es en un sistema, del sistema. El ejemplo que toma, el de los indios americanos ante el colonizador español, es clarificador: simulan aceptar las reglas, lo que deriva que, en tanto simulación, es un rechazo del sistema social colonial por parte del sistema social indígena. Las consecuencias prácticas de tal simulación parecen borrosas, como un esfuerzo un tanto inútil, salvo como resistencia simbólica - lo que puede decirse, generalizando, de los mecanismos de resistencia propuestos por de Certeau - siendo aquí lo importante su lugar fronterizo, entre la aceptación y el rechazo, entre el ser y el no ser.

Es precisamente el lugar interfronterizo o transfronterizo y, en definitiva, efímero — «lo que se gana, no se guarda», con la táctica- lo que atrae a de Certeau al concepto de táctica, que le lleva a reivindicarlo sobre el de estrategia. Es el concepto que permite a este autor situarse en una perspectiva crítica: «la táctica está determinada por la ausencia de poder como la estrategia está organizada por el postulado de un poder» (de Certeau, 1990:62). Las tácticas se configuran como un ejercicio - no consciente- de resistencia, de aquí su movilidad, pues son resistencia frente a la alteridad y su movimiento depende de los movimientos del otro. Una perspectiva crítica que le sitúa en el campo intelectual 
como crítico de los críticos. El concepto de táctica, como mecanismo de resistencia, se opone al de dispositivos de control de Foucault. Ambos son del detalle, de la micropolítica, pero en sentidos inversos con relación al poder. Mientras que con respecto al concepto de estrategias de Bourdieu, que asume en buena parte, enfrenta la propia presencia de las tácticas. Las estrategias - imaginarias, proyectivas - encuentran su resistencia en tácticas.

Con relación al tiempo, las estrategias conllevan perduración en el tiempo. Son del orden de la duración. Las estrategias temporales son proyecciones en el tiempo, orientaciones en el tiempo. Orientaciones en el tiempo desde un espacio simbólico, como subraya de Certeau. Temporalmente pueden ser imaginarias, pero hechas desde un lugar simbólico, desde una posición en el sistema social. Ahora bien, en su temporalidad más o menos imaginaria exigen la asunción de:

- Estabilidad de sentido, que puede aceptarse como producto de estabilidad de la estructura social o estabilidad sistémica, del sistema social.

- Horizonte, que se configura desde una doble cara, como: a) un límite de tal proyección, hacia el donde hay que llegar; b) proyección que marca ritmos, en la medida que se perciba cerca o lejos ese horizonte y las capacidades para llegar, así se dan estrategias del corredor de fondo y estrategias de la proximidad, de lo asequible.

- Todo sistema sitúa a sus elementos en función de su capacidad estratégica: con futuro o gran capacidad estratégica, anclados en el pasado o sin futuro.

Desde este punto de vista, las estrategias pueden interpretarse insertas en la lógica propia de los sistemas sociales.

Michel de Certeau subraya la negatividad de las tácticas con respecto a las estrategias. Como si fueran su reverso. Cuestión distinta es la articulación entre ambos conceptos, donde cabe concebir las tácticas como agujero, ruptura o quiebra de las estrategias, en cuanto huecos en la estrategia. La linealidad de la estrategia se quiebra en las tácticas, como las estrategias de las mujeres cuadros técnicos se quiebran abrumadas por las tácticas de dar salida a las demandas como responsables primeras de sus hijos menores. Y como huecos en el sistema social del que son el centro, al ponerse en comunicación con otro sistema social. Las tácticas son la negación de las estrategias; pero, también, algo más: la negación que, a su vez, permite estrategias en el sistema social. Por ello, en la relación entre tácticas y estrategias cabe hablar de negación dialéctica y, por lo tanto, de relación dialéctica. Con las tácticas, se intenta superar la resistencia a la imposición temporal de las estrategias. Así, por ejemplo, para obtener la muestra diseñada (del orden de las estrategias) se negocian nuevas visitas, otras citas, con los entrevistados, superando sus negativas. En esta superación, se incorpora parcialmente el tiempo de los observados para integrarlo en el tiempo de la observación. La vida cotidiana de los observados empíricamente se convierte en el entorno del sistema social ciencia. Por lo tanto, más que de oposición entre tácticas y estrategias, en la observación empírica se apunta cierta articulación. Al menos, una relación dialéctica. Es cierto que la temporalidad de la observación

EMPIRIA. Revista de Metodología de Ciencias Sociales. N. ${ }^{2} 23$, enero-junio, 2012, pp. 137-161. ISSN: $1139-5737$ 
acaba imponiéndose - la cita con los observados, cuando ellos pueden, no puede llevarse a cabo fuera del tiempo de la observación. Por lo tanto, hay un límite a la negociación de temporalidades, pues el sistema observador tiene límites temporales.

En las estrategias, hay un sujeto de querer y de poder. Relativizado por Bourdieu en una red de reglas, de fuerzas, de lógicas, quedando en un segundo plano la voluntad de ese sujeto. Combate el dominio de una perspectiva en clave de las cosas de la lógica, para sustituirla por una en clave de la lógica de las cosas (Bourdieu, 1984). Pero incluso en el caso de de Certeau cabría preguntarse para qué el sujeto. ¿Para dar consistencia a las tácticas, darles una especie de soporte, de dar cuerpo a los mecanismos de resistencia? Más bien parece al contrario: son las tácticas las que dan consistencia a los sujetos. Las que constituyen sujetos. Especialmente a sujetos dominados de los que habla de Certeau. Lo que observa empíricamente el historiador francés es la ausencia de una supuesta normalidad, estandarización u homogeneidad en las prácticas. A partir de aquí, sitúa como origen de tales desvíos a los sujetos, al poder y querer de los sujetos, cuando tal querer y poder se sitúa en las estrategias. ¿Dónde observa empíricamente este poder y querer? En el reverso de la lectura de Vigilar y castigar de Foucault, como reverso de los dispositivos de poder. En Foucault, el sistema punitivo se comunica con el sistema educativo, generando dispositivos, tácticas del poder. De Certeau, subraya cómo las tácticas también funcionan como resistencias en esa comunicación. A partir de las tácticas de Michel de Certeau, la comunicación entre sistemas sociales no indica un dominio sino una continua negociación, de aquí que quepa concebir las tácticas como un esfuerzo de la comunicación y, por lo tanto, como comunicaciones forzadas.

Desde el punto de vista de los sujetos, las estrategias pueden entenderse como una ordenación del tiempo que ordena sujetos. El margen para las estrategias viene dado por la posición de los sujetos (Bourdieu, 1998:140 y ss.). Ahora bien ¿qué es una posición en la estructura social? Un lugar en la lógica del sistema social.

Por último, en la justificación de una interpretación sistémica de los conceptos de estrategia y táctica cabe una mínima reflexión sobre el papel del concepto mundo de vida. En principio, el mundo es simultáneo. El mundo es el mundo de todo lo simultáneo. Es de lo presente. Algo que puede proyectarse en el entorno del sistema. Admitámoslo como nicho de comunicación entre sujetos, como si los sujetos encontrasen un espacio regresivo. Como espacio regresivo es donde mayor margen hay para las tácticas, según la concepción de de Certeau, para los instantes. En cuanto espacio privado, toda rebeldía es casi posible; mientras siga ceñida al nicho. Todo mecanismo de resistencia es posible. Ahora bien, es donde se comunican los sistemas, de ahí que fluya la comunicación, comunicación entre sistemas. De hecho, una de las comunicaciones más difíciles se establece con el sistema de observación.

El observador es un mundane reasoner que pone en cuestión la base de los mundos de vida. Una intersubjetividad que pone en cuestión los métodos con 
que se intenta observar la experiencia de ese mundo, informarse sobre él. La observación rompe un espacio regresivo construido frente a la ingerencia y la observación, como espacio privado. La observación hace público el espacio privado y, lo más importante, hace tiempo público un tiempo privado. Y las rompe precisamente de manera temporal. Lo que lleva a plantearlo en clave de intersubjetividad y no de relación entre sistemas ${ }^{5}$.

\section{LA OBSERVACIÓN EMPÍRICA A PARTIR DEL TIEMPO}

Hay una simultaneidad que exige una previa disposición de tiempos. Disposición de tiempos para la observación empírica concreta. Desde el sistema observador, se dispone el tiempo de la observación en un horizonte de planificación en el que cabe distinguir niveles:

- Disponibilidad del tiempo de la observación, proyectado en los plazos y en el cronograma, en la gestión de la investigación como conjunto, haciéndose manifiesta la dimensión más estratégica, en clave de de Certeau (1990), de la temporalidad de la observación, tomando como criterio preferente su racionalización e incluso su eficiencia, cuando se vincula el tiempo con coste económico. Dentro de la investigación empírica, es el cronograma el que muestra con mayor fuerza la duración como realidad del orden (Bachelard, 2001:20), como vivencia que ordena.

- Disponibilidad del tiempo para el campo de la observación, para situar en el campo - valga nuevamente como recurso metafórico el de la fotografía - al fenómeno observado. Especialmente para situar en el campo a los sujetos observados, reconvertidos en objetos para la observación (el tiempo de los sujetos observadores tiende a diluirse, a dejarse de tener en cuenta: desaparece del campo). ¿Qué tiempo se puede disponer de los observados? Entonces, se desciende a decisiones que especialmente parten de un doble criterio:

- De la disponibilidad de tiempo de las distintas categorías sociales. Se asume que no todas las categorías sociales disponen del mismo tiempo para ser observadas, ni tienen los mismos horarios para tal disponibilidad: disponibilidad restrictiva para las categorías dominantes; flexible y amplia para las categorías dominadas. El grado de disponibilidad aparece así proporcionalmente inverso a la posición de dominación. Tampoco es igualmente disponible todo el tiempo de las jornadas, de la semana o del año. Cuestiones que hay que tener en cuenta a la hora de diseñar el trabajo de campo y hace que la probabilidad de ser

5 Tal discusión del concepto de mundo de vida tiene por consecuencia un replanteamiento del concepto de mundo y su relación con la comunicación, especialmente fijándonos en la producción comunicativa del mundo: «El mundo no es lo que la comunicación participa; más bien la comunicación lo divide en lo que ella participa y lo que no participa» (Luhmann, 1996:25). El mundo de la vida está más allá de la comunicación. Es sobre lo que la comunicación opera. 
observado sea inversamente proporcional a la posición en la estructura social ${ }^{6}$. Desde este punto de vista, la posición en el espacio social se explica sobre todo en clave de tiempo (Bourdieu, 1984:4). Es una posición en el tiempo social. Una posición que tiene en cuenta la observación empírica.

- De la tradición —o grado de estandarización o formalización — vinculado a unas prácticas de investigación social a las que se otorga una especie de duración media y, sobre todo, límites en la disponibilidad del tiempo de los observados para ser sometidos a la observación: veinte minutos para la entrevista con cuestionario estandarizado aplicado telefónicamente, dos horas para el grupo de discusión, un año para la observación participante en una comunidad cerrada y lejana. Es como si las prácticas pusiesen en juego una disponibilidad que les es propia.

Aun cuando en esta disponibilidad para el campo no puede dejarse de ver también su dimensión estratégica, se asume que el propio campo es un escenario de decisiones, incluso de reacciones tácticas, de negociaciones tácticas. Por ello, apenas se programan en el proyecto. Quedan para decisiones posteriores: cuando se tenga más perfilado el diseño, cuando se sepa, cuando se hable con los potenciales informantes, después de un estudio piloto que nos diga el tiempo que lleva aplicar un cuestionario, etc.; y a la vez, quedan encuadradas las indecisiones previas en la planificación estratégica del cronograma. A lo sumo, en las prácticas de observación empírica más estandarizadas - como la encuesta con cuestionario estandarizado- se realizan estudios pilotos para controlar, entre otras cosas, el tiempo de simultaneidad de disponibilidad de temporalidades, del encuentro.

Se establece un hueco en el tiempo del cronograma para concretar estas decisiones tácticas; pero no se adelantan tales decisiones. El cronograma proyecta una sucesión de resultados en el tiempo y, sobre todo, en el calendario. Sitúa el trabajo de campo en la temporalidad social. En el cronograma está la duración que niega lo instantáneo, como si negando la espontaneidad se pudiese negar la instantaneidad.

Las tácticas tienden a desaparecer de las escrituras de la investigación empírica, de los relatos de la investigación. Tanto del proyecto, sin lugar en el referido cronograma, como en los informes. Quedan, a lo sumo, de manera prevista e implícita en los manuales de campo, para los entrevistadores ${ }^{7}$, o en el informe de incidencias de campo, para la encuesta con cuestionario estandarizado, o en los diarios de campo de la observación participante, envueltas en el conjunto de la más o menos literaria descripción de la investigación y con más proyección en

\footnotetext{
${ }^{6}$ Así, las amas de casa en exclusividad, han sido el principal recurso de la investigación de mercado y consumo. Se justificaba por su posición privilegiada en los procesos de decisión de compra. Sin embargo, el peso de su disponibilidad temporal para la observación es relevante.

${ }^{7}$ Conjunto de instrucciones que se redactan para cada encuesta con cuestionario estandarizado con normas para los entrevistadores.
} 
un tipo de observación de la observación, como es la autorreflexión y control de la propia investigación (García Jorba, 2000), que para otras concreciones menos privadas de la observación de la observación.

Las referencias a las tácticas alcanzan sólo registros habitualmente ausentes del espacio público, de la observación más general de la observación, como ocurre cuando se llega a la publicación de la investigación en forma de artículo o monografía. Parece reservada su existencia pública y publicada a los manuales de metodología, a esa literatura más o menos normativa del deber ser de las prácticas de investigación, incluyendo su deber ser temporal. Y ello con notable precaución, como muestra, por ejemplo, la ausencia a referencias temporales sobre la duración de la aplicación de los cuestionarios. Los momentos tácticos tienden a silenciarse en las referencias metodológicas de las investigaciones, salvo en monografías que, desde un enfoque eminentemente metodológico, analizan el registro de los tiempos de observación como incidencia (Díaz de Rada y Núñez, 2008).

Sin embargo, en las referencias a la observación empírica, hay una retórica de la duración. Asimilada al esfuerzo de observador y en menor grado al esfuerzo de los observados. Se habla de tantos años de investigación, de la acumulación de horas o meses en un laboratorio o en una comunidad, realizando observación participante. Desde este punto de vista, los observadores participantes son los que en mayor medida tienden a convertirse en autores (Geertz, 1989) y héroes de la duración en la observación ${ }^{8}$. Por el contrario, la duración de lo observado aparece absorbida en la duración de la observación. Es precisamente a través de la duración, como la observación de la denominada observación participante se impone a lo observado. Integra lo observado desde un estar ahí, frente a ello, como si se encontrase en el sistema observado. La participación de la observación participante es, sobre todo, una participación temporal. Se participa del tiempo de los observados, siendo la excepción, entre las prácticas de investigación social, a la imposición de la temporalidad de la observación a las temporalidades de los observados. Y en la observación, en los registros de la observación — desde el cuaderno de campo, al informe- sólo quedarán las duraciones. ¿Dónde quedan esas repentinas imágenes del trabajo de campo, a partir de las que parece cambiar todo lo visto, que abren intuiciones?

Incluso en la observación participante, los instantes, en los que se traza la distinción entre un antes y un después, quedan sin registrar. El instante rompe la investigación o, como decía Ibáñez siguiendo la teoría de las catástrofes, rompe y hace al investigador. La catástrofe, se produce en un instante, sin duración. En la duración, por otro lado, se niega el instante.

La duración da unidad (Bachelard, 1992:18). Da unidad a la observación empírica: tantas horas de observación participante en tal comunidad, tantas horas grabadas de entrevistas o grupos de discusión, etc. Desde la unidad, la duración se recupera. Sin embargo, en la propia observación empírica tiende a con-

${ }^{8}$ Como contrapunto en clave de humor, están los imprescindibles textos de Nigel Barley (1989; 1993).

EMPIRIA. Revista de Metodología de Ciencias Sociales. N. 23, enero-junio, 2012, pp. 137-161. ISSN: $1139-5737$ 
vertirse en una especie de losa, que parece convenir dejar en la caja negra de la observación. Así, por mencionar sólo unos ejemplos: a) se toma como indicador del éxito de la observación empírica en ciencias sociales que el momento de la observación les parezca corto a los observados, se asimila entonces a una reactividad leve, menor; b) por el contrario, si la duración de la observación empírica se convierte en tema de la observación - como puede pasar en la aplicación de un cuestionario o la realización de una entrevista en profundidad o un grupo de discusión - se tiene por problema; c) la dificultad que tienen quienes se estrenan en la investigación empírica, como los estudiantes de ciencias sociales, para hacer durar sus encuentros empíricos con los observados, quedando la duración como problema. Todos estos ejemplos ponen de manifiesto la configuración de la duración como una losa en el momento, en el instante, de la observación. Es como si se quisiese hacer investigación sin duración o limitarse a recoger ésta como sinónimo de un esfuerzo que busca reconocimiento. Salvo en la relativa excepción de la observación participante, la duración es un atributo negativo de la observación empírica.

La observación empírica, al menos en lo que respecta al contacto con los otros, parece tender a una duración cero (0), al instante, como si así desapareciese la observación y, desde un punto de vista material, el esfuerzo de la observación. Recomendación de cuestionarios cortos, entrevistas cortas y a una elusión de la previsión de duración del encuentro con los otros según se va de la formalización de la perspectiva cuantitativa a la perspectiva cualitativa de investigación social. Una losa de la duración que es mayor cuando se impone en prácticas que se suponen previamente abiertas. Es aquí donde merece la pena comentar la creciente temporalización que, a través de los guiones, se lleva a cabo en algunas prácticas cualitativas de investigación social, como el grupo de discusión. Lo que tiene de interesante esta práctica es la apertura al suceso, al instante, que es todo lo contrario a la duración. ¿De qué sirve dar un tiempo para que el grupo hable sobre un tema? Algo que sólo es comprensible desde una investigación que tiene ese encuentro, la situación grupal, más como dispositivo para hacer hablar - aunque sea mal, aunque sea rápido- que para esperar los discursos grupales, con su ritmo. Los guiones de dinámicas de grupos de discusión o grupos focalizados fuertemente prefijados temporalmente someten previamente los movimientos tácticos de los observados, dejándolos entre la reacción (reactividad) inmediata o el silencio (dificultades para que surja algo más que la respuesta buscada); pero, para que la práctica de investigación funcione, son soliviantados, transgredidos. Si lo que se impone es éste tipo de guión es a costa de la potencialidad de la práctica de investigación.

La fijación previa de la duración, como la propia duración, de la observación empírica se vuelve contra la propia observación empírica. Así se tiene el significado de la maduración (Campbell y Stanley, 1973), de los observados como observados, como algo a evitar. Parece que el objetivo tiende a una duración cero, al instante. Pero ¿qué es lo que se intenta evitar con la maduración? Se dice que se intenta evitar que los observados aprendan, que adquieran conciencia de 
lo que se quiere observar y, entonces, adapten sus comportamientos o respuestas a lo requerido. Con la evitación de la maduración, que es consecuencia de la mera duración de la observación empírica, se intenta evitar que los observados sepan de la observación más que los observadores. Evitar, en definitiva, que pasen del sistema observado al sistema observador.

\section{LOS SISTEMAS SOCIALES OBSERVADOS: A PARTIR DEL TIEMPO, LAS ESTRATEGIAS Y LAS TÁCTICAS}

Como se ha visto, la observación empírica tiende a una duración cero, al instante. Sin embargo, todo sistema tiende, por el contrario, a la duración. Es duración. Cuestión que genera la cuestión sobre la posibilidad de que el instante recoja la duración en una sociología del tiempo. Nos aproximamos ahora a la teoría de sistemas, especialmente de la mano de Luhmann, en cuanto aporta una plausible descripción sociológica de cómo se entrecruza la temporalidad en la observación. Es más, el tiempo conforma los sistemas sociales, diferenciándolos del entorno y otros sistemas. La cuestión es ¿cómo pueden relacionarse en el tiempo sistemas distintos, como ocurre en la observación empírica, si constituyen lógicas temporales distintas?

Siguiendo de cerca autores como Luhmann (2007:105), la sociedad es un sistema complejo necesitado de tiempo. Como sistema complejo: compuesto de relaciones. Algo que alcanza descripciones como que son las relaciones sociales las que necesitan tiempo, más allá de los individuos que forman parte de tales relaciones. El lugar que cabe dar a los conceptos de estrategia y táctica en la racionalizada concepción sistémica es el siguiente:

- Estrategia como diferencia y diferenciación, como rutas, trayectorias y proyecciones en un determinado sistema social. Estrategias del sistema social, como diferenciación del propio sistema social. Diferenciación temporal, que exige duración. Como proyección, es imaginaria. Como es el propio concepto de operación que sólo adquiere sentido como productora de sentido. La estrategia es una operación del sistema, en conexión con otras operaciones: «cada sistema tiene su propia memoria, organiza sus propias anticipaciones en conexión con sus respectivas operaciones» (Luhmann, 1996:33), incluyendo así la estrategia en la producción de sentido. La observación sobre el sistema social ciencia se ha centrado especialmente en esta temporalidad, en la situación de las operaciones del sistema con relación a un pasado que «supera» y un futuro que «construye» como horizonte. Buena parte de la filosofía moderna y la sociología de la ciencia ha trabajado sobre estos aspectos desde una perspectiva macroestructural ${ }^{9}$.

\footnotetext{
${ }_{9}$ Seguramente el ejemplo que mejor ilustra esta perspectiva es la propuesta de Kuhn (2005), extensamente referido en las aulas.
} 
- Táctica como reacción, en buena medida fruto de la repetición, lo que incluye las condiciones y posibilidades de la repetición, derivada de rutinas. La repetición como control del ruido: la economía del tiempo en la relación entre sistemas. En el instante. Como instante en el presente, es real. La observación sobre el sistema social ciencia, especialmente cuando se observan sistemas sociales, ha prestado bastante menos atención a esta temporalidad, cuya distinción concentra el esfuerzo de este trabajo.

Cada sistema, en la distinción funcional entre sistemas, se comunica con otros, se encuentra con otros sistemas en una adaptación continua que puede comprenderse en clave de tácticas. Participa con otros sistemas en las tácticas, constituyéndose respectivamente en entornos: «Para descubrir la relación entre conciencia y comunicación hemos elegido en un principio una formulación de la teoría de sistemas; es decir, hemos observado que cada uno de esos sistemas constituye el entorno del otro. Es posible iniciar otros análisis tomando en consideración al factor tiempo» [...] «La comunicación y la conciencia sólo pueden operar sincrónicamente, de igual modo que el sistema sólo existe de manera simultánea al entorno, no antes ni después» (Luhmann, 1996: 47).Pero se distingue entre sistemas por las estrategias.

Según Luhmann, no puede hablarse de adaptación de un sistema a un entorno (Luhmann, 2007:27). Menos aún entre sistemas. Habla de acoplamiento y procesamiento. Un acoplamiento que incluye la temporalidad. Un acoplamiento de temporalidades de sistemas en la observación

La comunicación entre sistemas, o cómo se configura uno en el entorno del otro es la clave para comprender ese encuentro entre distintos sistemas sociales que supone la observación empírica: «En la medida que se comunican todos los sistemas parciales participan de la sociedad; en la medida en que comunican de modo distinto, se distinguen entre sí» (Luhmann, 2007:112). En la medida que se comunican (tácticas), funcionan. En la medida que se comunican de modo distinto (estrategias), se distinguen entre sí. Pero la propia comunicación entre sistemas alimenta la distinción: «el concepto de acoplamiento estructural nos recuerda igualmente que el sistema registra de manera permanente irritaciones provenientes del entorno, tomándolas como punto de partida para la reespecificación de sus propias estructuras» (Luhmann, 1996: 27). El sistema observador registra como irritación su acoplamiento a las temporalidades del sistema observado.

Evidentemente, Luhmann no utiliza los conceptos tácticas o estrategias para dar cuenta de estos procesos. El más parecido al primero es el de acoplamiento, donde lo más importante es el rasgo de simultaneidad que incorpora, netamente temporal:

«El concepto de acoplamiento estructural designa una relación de simultaneidad, pero no designa, por lo tanto, ninguna relación causal» (Luhmann, 1996: 35). En el estudio empírico sobre el tiempo, se vio la imposibilidad de la simultaneidad entre sistemas. Ello a pesar de que algunas lo intentan denodadamente y a pesar de los obstáculos de todas las estructuras. La simultaneidad se da entre sistemas, no en los sujetos. Ni siquiera en los sistemas. 
La distinción entre sistemas es una distinción entre temporalidades. De aquí que un acoplamiento entre sistemas, como es el que supone la observación, exige un acoplamiento temporal. El sistema de la observación tiene su temporalidad. El sistema del «real-world» tiene su temporalidad ¿cómo se articulan estas dos temporalidades?

\section{LAS TEMPORALIDADES DEL SISTEMA SOCIAL CIENCIA}

El sistema ciencia se caracteriza por una operación: reducción. Operación también aplicable al tiempo. Reduce el tiempo real, el tiempo observado en el tiempo de la observación. Si el tiempo real, por mucho que haya que preservarlo, no queda subsumido en el tiempo de la observación y, por tanto, en sus estrategias temporales, no puede hablarse de observación. El tiempo de la observación tiende a comprender el tiempo observado, generando una reducción en éste. Es más, el tiempo de los sistemas deriva de su observación: «la existencia de estos sistemas se da solamente en el momento. El resto es únicamente susceptible de observación, e inclusive esto sólo de manera operativa, es decir, nuevamente, sólo en el momento correspondiente de la operación llamada observar» (Luhmann, 1996:32). El tiempo de lo observado se configura dentro del sistema de observación; pero dentro de unos límites. Una reducción que puede entenderse como simulación: «El investigador tiene la opción de reducir el tiempo real, creando un tiempo experimental, o combinando esas dos escalas de tiempo de alguna manera, como el contexto temporal por el desarrollo de los acontecimientos durante la simulación» (Kelly y McGrath, 1988:36).

Para aproximarnos a la subsunción del tiempo observado en el tiempo de observación en la observación empírica, hay que establecerse, en la medida de lo imposible, en el acoplamiento entre sistemas, siguiendo la propuesta teórica de Luhmann. Pero antes, veremos cuales son las temporalidades del sistema social ciencia. Es decir, las temporalidades que funcionalmente lo distinguen.

El sistema ciencia, en cuanto observación de la observación, se fundamenta en una especial temporalidad antes/después. Lo nuevo marca el tiempo en el sistema social ciencia (y de paso, en la modernidad). Temporalidad que se encuentra en el análisis de la causalidad —en el modelo bacon/humeano- donde la causa ha de ir antes que el efecto (Kelly y McGrath, 1988:16).

Teniendo ahora en cuenta las disponibilidades del tiempo y las disposiciones con respecto al tiempo de la observación empírica, cabe distinguir los siguientes niveles en la temporalidad de la práctica del sistema ciencia con la finalidad de acceder a su temporalidad fundamental antes/después. Son estos niveles:

- Los niveles de la disposición del tiempo en la observación, que derivan de una directa proyección de las temporalidades del sistema observado, y son eminentemente teóricos:

- Nivel de la configuración del tiempo del sistema observado como anterioridad, como pasado, como antes. Tiende a configurarse en clave 
de los antecedentes de la observación, como antecedentes de observación - teórica o empírica- del sistema observado.

- Nivel del horizonte de observación, que se sitúa en el después de la observación, en los logros de la observación, en lo que explica. Dispone el tiempo en presente, como ocurre en los llamados estudios descriptivos, o en futuro condicional, como ocurre cuando el horizonte de investigación es la causalidad.

- Los niveles de la disponibilidad que, como se ha señalado antes, llevan el sistema de la ciencia a la observación empírica:

- Disponibilidad de tiempo de la observación

- Disponibilidad del tiempo para el campo de la observación

En cuanto centrados en la observación empírica, adquieren aquí principal relevancia los niveles de disponibilidad temporal. ¿Cuál es su relación con los otros niveles? Desde el punto de vista táctico (cuestionarios cortos, entrevistas cortas) hay una inclinación de la observación empírica por hacer desaparecer la duración. La observación de la observación observa instantes desde el instante: «se puede observar en otro instante temporal en caso de que en este instante aún sea reconstruible lo que el observador observado habría observado en otro momento» (Luhmann, 1996:83).

Parece que, como subraya Bachelard (1992), el conocimiento se produce en un instante. Después, el compromiso integrará los instantes en duraciones. El trabajo sobre la observación, como la observación de la observación, dura, tiene duración; pero las duraciones, niegan el instante. El instante de una frase en un grupo de discusión, de una relación en el análisis de una tabla estadística, quedan negados en la reflexión que producen, en el trabajo secundario, en su reelaboración. Estructura análoga a la que analiza Freud en La interpretación de los sueños: la conexión del sistema inconsciente con el sistema consciente es del instante, mientras que la reelaboración, incluso la onírica, es de la duración.

La duración corresponde a impresiones esencialmente secundarias e indirectas (Bachelard, 1992:48), cuando la observación se inclina como observación de la observación. El instante, que no tiene duración en su seno, es la observación directa. Las estrategias no son de ese instante: «las estrategias de investigación se refieren a esos escenarios generales en los que la investigación puede ser conducida» (Kelly y McGrath, 1988:30).

El sistema de la ciencia necesita el acoplamiento estructural con otros sistemas, los observados. Tanto un sistema como otro presentan patrones temporales, integrados en sus respectivas estrategias, que suponen límites a las tácticas. Por ejemplo: la visita del encuestador a una persona que se le ha mandado una carta anunciándole que ha sido seleccionada en un muestreo nominal ha de hacerse pocos días tras la emisión de ésta; la próxima llamada al seleccionado para ser entrevistado no puede retrasarse indefinidamente; la observación de una comunicación, que ha de tener en cuenta varias generaciones y los cambios generaciones, tienen a no superar la vida del observador. Los acoplamientos tácticos tienden a tener su límite derivado de su subordinación a las estrategias de los sis- 
temas. Desde este punto de vista, el del sistema de la ciencia, la estrategia de observación tiene un lugar dominante: la duración del periodo del sistema de acción que ha de ser estudiado está temporalmente limitado por la extensión del período de observación (Kelly y McGrath, 1988:35). El sistema observador se impone al sistema observado.

\section{LA OBSERVACIÓN EMPÍRICA COMO SIMULTANEIDAD}

La operación de conocimiento fundamental es la observación. Toda observación empírica es observación de un suceder, de un suceso. Un suceso que pertenece simultáneamente a varios sistemas sociales, en el que se comunican sistemas sociales. Observar es una operación comunicativa; y la comunicación es una operación básica, genuinamente social (Luhmann, 2007:57). Plenamente social, podríamos decir, en la que la sociedad se hace presente. Por ello, todo suceso sucede en el presente, de manera simultánea: «El tiempo simplemente virtual del pasado y del futuro está presente en cada presente -aun si para el tiempo virtual lo simultáneo es algo completamente distinto que para el presente. Precisamente esta ilusión de la cultura escrita a la cual nosotros ya estamos acostumbrados, hace difícil recuperar la idea fundamental de que todo lo que sucede, sucede en el presente y de manera simultánea» (Luhmann, 2007:205).

En la situación de observación empírica misma, no puede hablarse de esa distinción temporal pasado, presente y futuro (pasado y futuro virtuales) creados por lo nuevo. Se pone en juego la ilusión de lo simultáneo. La ilusión de lo simultáneo viene de la mano de la simulación y del simulacro de la reproducción como real. Simulación de prácticas en el experimento, simulación de encuentros cotidianos - en el mundo de vida de los entrevistados - en las entrevistas, simulación de temporalidad discursiva en los grupos de discusión. La simulación es de las tácticas. Es más, cabe hablar de tácticas de simulación temporal: temporalidad para la producción discursiva en grupos de discusión y entrevistas, como si se pudiera decir todo en esos encuentros; temporalidad de tareas en los experimentos tan queridos por la psicología social.

Ilusión de lo simultáneo en un doble sentido. Por su reducción a instantes, que llegan a hacer de la temporalidad una ilusión. Porque la observación introduce un tiempo «artificial» en lo observado, el tiempo de la observación, como ocurre en el análisis de la causalidad: «el experimentador ha modificado el orden natural de al menos un importante conjunto de acontecimientos al introducir la manipulación de la variable independiente en el sistema» (Kelly y McGrath, 1988:35).

El suceso de la observación es un instante porque pertenece a varios sistemas, por exigencias de su simultaneidad. La observación es un acoplamiento entre sistemas: «La comunicación y la conciencia sólo pueden operar sincrónicamente, de igual modo que el sistema sólo actúa de manera simultánea al

EMPIRIA. Revista de Metodología de Ciencias Sociales. N. ${ }^{\circ}$ 23, enero-junio, 2012, pp. 137-161. ISSN: $1139-5737$ 
entorno, no antes ni después» (Luhmann, 1996:47). En la observación empírica se establece un acoplamiento de temporalidades de sistemas. Como se veía más arriba, el sistema observador (ciencia) ha de acoplarse a la temporalidad del mundo de vida de los observados (vida cotidiana), que actúa como entorno con respecto a aquél. El mundo de vida de los observados se ve alterado en su temporalidad por la exigencia de acoplarse al sistema observador, que actúa como entorno con respecto a aquél.

El esfuerzo táctico es del acoplamiento, del instante y, sobre todo, lo simultáneo. De hecho, la táctica es lo que define lo simultáneo: «el acoplamiento estructural define la extensión de lo que ha de aceptarse como simultáneo» (Luhmann, 1996:47). Ahora bien, precisamente porque la relación entre sistemas es de la simultaneidad, no cabe la causalidad: «El concepto de acoplamiento estructural designa una relación de simultaneidad, pero no designa, por lo tanto, ninguna relación causal» (Luhmann, 1996:35). La causalidad es propia del sistema social ciencia, de su función diferencial.

La simultaneidad genera irritaciones, sucesos. Incluso la ilusión de simultaneidad. La simultaneidad es lo importante en la observación cuando se trata de analizar los efectos de la observación en lo observado. Es la producción de la observación.

Lo que se observa, en esa reflexión sobre la producción de la observación, son tácticas, la comunicación entre sistemas que es el suceso de la observación empírica. Podría hablarse de observación de las consecuencias tácticas. Las estrategias se alimentan así de las tácticas.

El suceso empírico de la observación aparece así como fundamentalmente un esfuerzo del acoplamiento táctico. Un fundamento táctico que será integrado en la observación, dentro del sistema de la observación. Por lo tanto, internado en las estrategias de la observación. En la observación de la observación por el sistema entra en la lógica de sus estrategias. No es necesaria ya la simultaneidad, sino, al contrario, la temporalización del antes y después: «Si se toma en serio la temporalidad operativa de los sistemas observadores como observación, uno se forma una idea acerca de una conquista evolutiva que podríamos designar como desimultaneización del mundo» (Luhmann, 1996:80). En las estrategias temporales se desimultaneiza el mundo.

¿Qué preguntarnos sobre ese tiempo del acoplamiento táctico? No es preguntarse sobre qué causa la observación en lo observado. Es más: «el interés en las causalidades puede conducir a minimizar el ámbito de las simultaneidades» (Luhmann, 1996:48). Hay una doble ruptura de la simultaneidad en el enfoque causal: un antes/después en el sistema observado, y una práctica de observación que, bajo el modelo del laboratorio (Latour, 1991) intenta hacer el vacío del tiempo y, por lo tanto, rompiendo el tiempo del sistema observado.

En las estrategias del sistema social ciencia, los acoplamientos tácticos son observados, por mor de la observación de la observación, y, por ende, racionalizados. Por lo tanto, no sólo las estrategias son racionales, como apunta de Certeau, sino que racionalizan las reacciones tácticas cuando forman parte del sis- 
tema social de la ciencia, cuando se proyectan linealmente en el tiempo del sistema social. Incluso son racionalizadas en sus primeras apariciones, en esos espacios entre lo privado y lo público de la investigación: en los manuales para los entrevistadores, en los informes de incidencias y, sobre todo, en los diarios de campo.

Las estrategias van a definir/redefinir/indefinir las reacciones tácticas haciéndolas desaparecer de la escritura de la investigación. Incluso una práctica tan inclinada a la retórica de la descripci n, como es la observación participante, las tácticas temporales quedan subsumidas en tiempo del fenómeno observado (por ejemplo, Wacquant, 2004).

En la observación de la observación, el sistema está en su lógica, en sus estrategias. No es necesaria la simultaneidad, sino lo contrario, la temporalización del antes y después en la que la simultaneidad es un fardo: «Si se toma en serio la temporalidad operativa de los sistemas observadores como observación, uno se forma una idea acerca de una conquista evolutiva que podríamos designar como desimultaneización del mundo» (Luhmann, 1996:80).

La introducción de nuevas tecnologías de la comunicación en la observación aparecen inclinadas a favorecer cierta simultaneidad: el teléfono, internet. Algunas tácticas de observación están más cerca de la simultaneidad —investigación de audiencia mediante un panel de audímetros en los aparatos (televisión, ordenador) - a costa de cortar en algún momento con esa simultaneidad: cuando se envían los datos al ordenador central. Es aquí donde pueden empezar a cobrar sentido. Hasta el momento, son sólo registros sin sentido. Los datos quedan, entonces, condensados y confirmados: «La forma bilateral de la condensación y confirmación se cuaja, por así decirlo en la forma del sentido: en la forma de un sector actual para cada caso, que garantiza la simultaneidad del mundo en el momento y que la presenta como inmenso horizonte de otras posibilidades que se pueden actualizar sólo selectivamente como una u otra. Sin la operación temporalmente reductiva de la observación, no surgiría la formación del sentido. Pero, evidentemente, también es válido lo contrario, el sentido es el medio a través del cual la observación obtiene su forma» (Luhmann, 1996:83). Subsumidas en la racionalidad, las tácticas se difuminan. Las tácticas, pertenecientes al sentido práctico — siguiendo a Bourdieu — desaparecen cuando se impone el sentido lógico o sentido formal. La observación acaba —si es que puede hablarse de acabar - subsumiendo las razones prácticas y el sentido práctico en la razón lógica de la propia observación. Como doble hélice.

Caben dos mínimos apuntes para reflexionar, dos líneas de cuestiones. En primer lugar, la proyección en la diferencia de perspectivas de la investigación social. La diferencial concreción de las tácticas en la perspectiva cualitativa y en la perspectiva cuantitativa. Por otro lado, cuando el fenómeno observado es el tiempo en distintos sistemas sociales.

Toda observación es una reducción ¿qué tipo de reducciones lleva la observación del tiempo? ¿reducciones temporales en la observación del tiempo? En la medida que el tiempo social es el objeto, cabe diferenciar aquí entre tiempo ob-

EMPIRIA. Revista de Metodología de Ciencias Sociales. N. ${ }^{\circ}$ 23, enero-junio, 2012, pp. 137-161. ISSN: $1139-5737$ 
servado y tiempo de la observación. Desde la perspectiva privilegiada aquí, entre tiempo del sistema observado y tiempo del sistema observación, de manera que se produce una reducción en el primero, en el tiempo del sistema observado, en función de las distintas lógicas (estratégicas) y las posibilidades (tácticas). Una reducción que tiene por finalidad producir cierta simultaneidad entre los dos sistemas. La simultaneidad que permita la observación. Al menos, la posibilidad de generar la ilusión de simultaneidad de lo simultáneo (Luhmann, 2007:295). Tal ilusión de simultaneidad, presente en todo esfuerzo de observación, se hace más patente cuando lo que se observa es el tiempo.

¿Qué simultaneidad se produce en la aplicación de un cuestionario de presupuestos temporales? ¿en un grupo de discusión sobre el tiempo? Se habla del tiempo, fuera del tiempo referido. El sistema observador, interrumpe su tiempo, para traerlos a su tiempo.

¿Y en el registro de la observación más o menos participante? En la observación de Lazarsfeld (1996) del tiempo en recorrer una calle por parte de los parados y los ocupados. La observación sólo era posible preguntando a los observados su condición con relación a la ocupación.

\section{CONCLUSIONES}

En el trabajo, se parte de la evidencia de que no se puede entender la observación empírica sociológica sin el tiempo y que, sin embargo, ha recibido relativamente poca atención tal dimensión, como si fuese un hecho dado. El tiempo, que atraviesa todas las relaciones sociales, también se encuentra en la relación social que constituye la observación empírica sociológica. Para el análisis de tal relación se han articulado dos perspectivas teóricas muy distintas, la sistémica de Luhmann, en cuanto sitúa la temporalidad en la propia definición de la observación, y la que, poniendo más el acento en el actor, se aproxima a los rasgos más concretos de las prácticas, entre los que se encuentra el tiempo, como la de Michel de Certeau. Hay toda una brillante línea auténticamente metodológica, en cuanto se plantea de manera directa las cuestiones de método, que trata de concordar la unidad de análisis - razonamiento de la observación - con el razonamiento que se utiliza en los asuntos cotidianos (Cicourel, 1982). Aquí se analiza cómo, teniendo el tiempo como referencia, la observación y el mundo observado constituyen lógicas distintas, imposibles de poner en común, pues no son equivalentes, a pesar del respectivo esfuerzo de acoplarse en clave de tácticas. Sin embargo, a pesar de ser lógica y temporalmente distintos o distintos en su lógica temporal, la observación empírica se produce y, por lo tanto y desde la perspectiva aquí tomada, cierto acoplamiento entre el sistema observador y el sistema observado.

Tras subrayar la relevancia que puede tener la observación del tiempo en clave de los conceptos estrategia y táctica en la observación empírica y, por lo tanto, como proyecto, una sociología empírica del tiempo y una sociología del 
tiempo de la observación empírica, se entra en los caminos teóricos que cabría tomar en tal horizonte. En primer lugar, las tácticas en la observación empírica se han establecido como comunicación entre sistemas sociales, en la que uno de los sistemas sociales es el sistema ciencia. De aquí, sus características temporales: presente, de lo instantáneo. En la medida que es comunicación entre sistemas (sistemas-entorno), el tiempo se configura como simultaneidad. Tal simultaneidad parece inscrita en acoplamientos tácticos. Las estrategias han quedado incrustadas en la lógica del sistema, de cada sistema. Las estrategias temporales se muestran linealmente en un solo sistema; mientras que los acoplamientos temporales en la observación empírica fungen sistemas, acoplan sistemas en el tiempo.

Las relaciones entre estrategias y tácticas fundamentan el conocimiento científico en cuanto conocimiento empírico, como observación empírica. Las tácticas como la comunicación que se produce en el presente, en la simultaneidad de la observación empírica, que suele tomar entre los científicos sociales la forma de trabajo de campo. Las estrategias, como reelaboradoras de esa comunicación. Cuestión que, apareciendo análoga a la pareja lógica del descubrimiento/lógica de la explicación, plantea como problema la relación entre ambas, entre tácticas y estrategias. Lo que puede decirse es que alguna articulación es presumible.

En esa articulación, como doble hélice de la observación empírica y del sistema social ciencia, las estrategias subsumen a las tácticas. Al menos, de dos maneras, ya sea denegándolas, dejando en la caja negra de la observación el tiempo de la observación, ya sea racionalizándolas en la observación de la observación. Los esfuerzos y reacciones tácticos cumplen su función alimentando las estrategias con registros y comunicación y, sin embargo, desaparecen. Asumirlos supone que lo observado es producto de la observación, que el tiempo de los observados es una reacción al tiempo de la observación, y, por ello, se pone entre paréntesis.

En la medida que el sistema ciencia es observación de la observación, quedan racionalizadas temporalmente las tácticas, haciéndolas desaparecer del tiempo de la investigación. Las temporalidades tácticas de la observación desaparecen de la observación en la racionalización estratégica. La táctica se concreta en el envés de las estrategias, incluidas las estrategias temporales, desapareciendo de su publicación. La doble hélice sólo funciona dejando ver únicamente un solo lado, el tiempo diseñado de las estrategias o el tiempo vivido de los acoplamientos tácticos, el tiempo de la investigación o el tiempo en la investigación; pero cabe apuntar el carácter dialéctico de su relación, ya que el resultado de toda investigación se alimenta de ambos tipos de tiempo, aun cuando unos se cuenten -y, por lo tanto, cuenten- más que otros en el sistema social de la ciencia.

Distinguida la simultaneidad como una de las temporalidades básicas que conforman la estructura de la observación empírica sociológica y teniendo en cuenta su especial carácter reactivo, como se ha subrayado especialmente al 
constituir el esfuerzo de simultaneidad como esfuerzo táctico, queda ahora analizar sus implicaciones en procesos concretos de investigación. Queda el cómo se acoplan, actuando como entorno, los distintos sistemas con el sistema social de la ciencia, ya sea en función de la lógica de los sistemas sociales observados, ya sea en función de las distintas operaciones del propio sistema social de la ciencia según los métodos, prácticas o técnicas de investigación social utilizadas. Queda concretar una sociología del tiempo de la observación empírica sociológica. Cuestión que supera el horizonte de este trabajo, dejándolo como reto para observaciones posteriores realizadas desde una perspectiva metodológica.

\section{REFERENCIAS}

Bachelard, G. (1992): L'intuition de l'instant, París, Stock (e.o. 1931).

BACHelard, G. (2001): La dialetique de la durée, París, Presses Universitarires de France (e.o. 1950).

Banks, M. (2001): Visual Methods in Social Research, Londres, Sage.

BARLEY, N. (1989): El antropólogo inocente, Barcelona, Anagrama.

BARLEY, N. (1993): Una plaga de orugas, Barcelona, Anagrama.

Bauman, Z. (2005): Liquid Life, Cambridge, Polity Press.

Bourdieu, P. (1980): Questions de sociologie, París, Minuit.

BouRdieu, P. (1984): «Espace social et genèse des "clases"», en Actes de la recherche en sciences sociales, vol. 53-53, junio 1984, pp. 3-14.

Bourdieu, P. (1988): El sentido práctico, Madrid, Taurus.

BouRdieu, P. (1998): La distinción, Madrid, Taurus.

Bourdieu, P. (2002): Le bal des célibataires, París, Seuil.

Callejo, J. (1995): La audiencia activa, Madrid, CIS-Siglo XXI.

CAllejo, J. (2004): «La práctica del consumo en Bourdieu: contra el formalismo y el populismo», en L. E. Alonso, E. Martín y J. L. Moreno (eds.):, Pierre Bourdieu, las herramientas del sociólogo, Madrid, Fundamentos.

CAmpbell, D. T., Stanley, J. (1973): Diseños experimentales y cuasiexperimentales en la investigación social, Buenos Aires, Amorrortu.

CeA D’AnCona, M. A. (1999): La metodología cuantitativa: estrategias y técnicas de investigación social, Madrid, Síntesis.

De Certeau, M. (1990): L'invention du quotidien. 1. Arts de faire, París, Gallimard.

De Certeau, M. (1993): Mai senza l'Altro, Milán, Fabbri Editori.

De Certeau, M., Giard, L., Mayol, P. (1994): L'invention du quotidien. 2. Habiter, cuisiner, París, Gallimard.

Cicourel, A. (1982): El método y la medida en sociología, Madrid, Editora Nacional (e.o. 1962).

Díaz de RADA IguzQuiza, V. y A. NúÑEz VILluendas (2008): Estudio de las incidencias en la investigación con encuestas. El caso de los barómetros del CIS, Madrid, Centro de Investigaciones Sociológicas.

Douglas, J. (1976): Investigative Social Research, Beverly Hills, Sage.

FLICK, U. (2004): Introducción a la investigación cualitativa, Madrid, Morata.

Foucault, M. (1978): Vigilar y castigar, Madrid, Siglo XXI. 
García Jorba, J. M. (2000): Diarios de campo, Madrid, Centro de Investigaciones Sociológicas.

GEERTZ, C. (1989): El antropólogo como autor, Barcelona, Paidós.

Kelly, J. R., McGrath, J. E. (1988): On Time and Method, Newbury Park (Calif.):, Sage.

KRISNHASWAMY, A. (2004): «Participatory Research. Strategies and Tools», Practitioner: Newsletter of the National Network of Forest Practitioners 22: 17-22.

KuHN, T. S. (2005): La estructura de las revoluciones científicas, México, Fondo de Cultura Económica (e.o. 1962).

LATOUR, B. (2001): Nunca hemos sido modernos, Madrid, Debate.

LAYDER, D. (1993): New strategies in Social Research, Cambridge, Polity Press.

LAZARsfeld, P. F., JAHOdA, M., ZeISEl, H. (1996): Los parados de Marienthal, Madrid, La Piqueta.

LóPez MarTínez, M. I., y Hernández SÁnchez, E. (2002): «Proyección diacrónica del Curso de Lingüística General», Tonos: Revista Electrónica de Estudios Filológicos, 4.

LuHMAnN, N. (1996): La ciencia de la sociedad, México-Barcelona, Universidad Iberoamericana-Anthropos.

LuHMAnN, N. (2007): La sociedad de la sociedad, México, Herder.

MAYO, E. (1975): Hawthorne and the Western Electric Company. The Social Problem of an Industrial Civilization, Londres, Routledge y Kegan Paul (e.o. 1949).

PRIETO, C. (2007): «Clases de sujetos, clases de actividades y clases de tiempos: una mirada desde la perspectiva del género», en C. Prieto (ed.):, Trabajo, género y tiempo social, Madrid, Hacer-UCM, pp. 9-20.

Saussure, F. (1983): Curso de lingüistica estructural, Madrid, Alianza.

Sutton, C. D. y D. MatThew (2011): Social Research. An Introduction, Londres, Sage.

TORNS, T. (2005): «De la imposible conciliación a los permanentes malos arreglos», Cuaderno de Relaciones Laborales, $\mathrm{n}^{\circ} 23$ (1).

VALLES, M. S. (1997): Técnicas cualitativas de investigación social, Madrid, Síntesis.

WACQUANT, L. (2004): Entre las cuerdas, Madrid, Alianza.

WebB, E., D. T. CAmpbell, D. Schwartz y L. Sechrest (1966): Unobtrusive Measures, Chicago, Rand McNally. 
